\title{
Hebammenbetreuung bei kindlichen Fehlbildungen: Gibt es den richtigen Weg?
}

Jelena Rensinghoff, Karin Weiß

Wenn Eltern durch vorgeburtliche Untersuchungen bereits in der Schwangerschaft die Diagnose bekommen, ihr Kind sei besonders, dann ist das immer ein Schock. Wir als Hebammen werden durch die zunehmende Rate an pränataldiagnostischen Untersuchungen immer häufiger damit konfrontiert. Wie können wir damit umgehen? Wie können wir die Eltern bestmöglich unterstützen? Und wo sind die Grenzen der Hebammenarbeit? Ein Leitfaden.

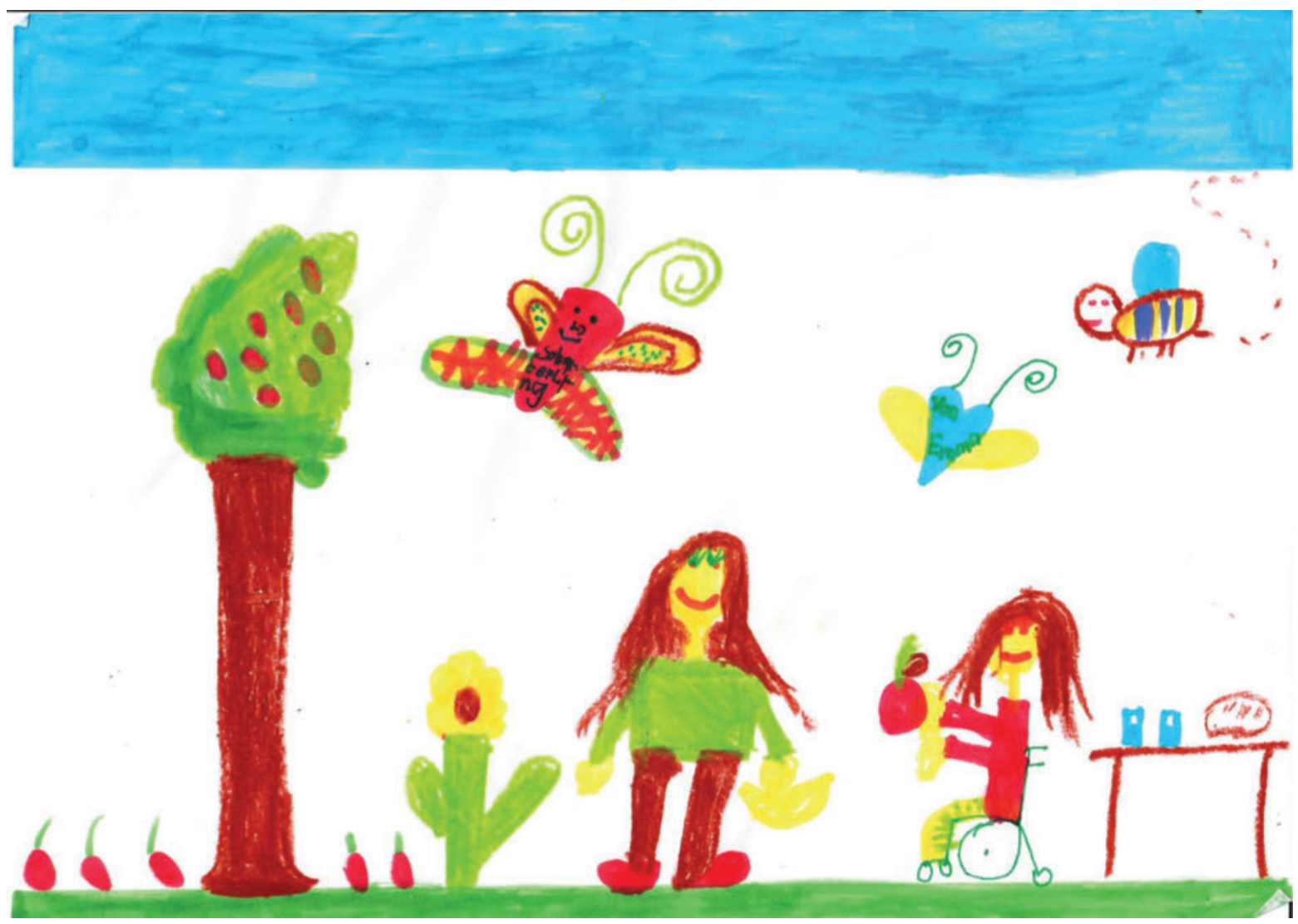

- Abb. 1 Auch aus den schwierigsten Entscheidungen des Lebens kann etwas Gutes erwachsen: Durch unsere Betreuung als Hebammen können wir Eltern ein Stück auf ihrem individuellen Weg begleiten und tragen so einen wichtigen Teil dazu bei, dass sie gestärkt aus der Lebenskrise hervorgehen. (Quelle: privat)

Die Rolle von Hebammen in der Schwangerenbetreuung, während der Geburt und im Wochenbett ist immer bedeutungsvoll. Wenn das Kind aber nun nicht kerngesund ist, wird die Unterstützung für die Eltern umso wichtiger und für uns teilweise zur Herausforderung. Gibt es da überhaupt einen richtigen Weg oder fühlt sich alles falsch an? 


\section{Präpartale Betreuung}

\section{Eine schwere Entscheidung}

Hebammenbetreuung beginnt im Idealfall im ersten Trimenon der Schwangerschaft und ist kontinuierlich. Nachdem eine Vertrauensbasis geschaffen wurde, ist es sinnvoll, bereits im Vorfeld über vorgeburtliche Untersuchungen und deren Durchführung zu sprechen und sich mit möglichen Konsequenzen auseinanderzusetzen [21][23]. Verschiedene Studien zeigen, dass sich die Eltern unter Zeitdruck fühlen, sobald ein positiver Befund vorliegt. Schnell soll eine Entscheidung über das ganze Leben getroffen werden, oft nach Tagen, manchmal sogar schon nach Stunden [3][18]. Auch der gesellschaftliche Handlungsdruck trägt zur Verzweiflung bei-wird doch hier und da suggeriert, dass das Leben mit einem behinderten Kind heutzutage vermeidbar gewesen wäre [22][21][18]. Die Angst vor Diskriminierung ist häufig ebenso präsent wie die Angst ein Leben mit einem eingeschränkten Kind nicht bewältigen zu können [3].

Durch die pränatale Diagnostik erhoffen sich die meisten Frauen die Bestätigung, dass ihr Kind gesund ist [6][21]. Doch was, wenn dem nicht so ist? Je nach Schweregrad der festgestellten Beeinträchtigung stellt sich die Frage, ob das Kind lebensfähig ist oder nicht. Und selbst unabhängig davon gilt es zu entscheiden:

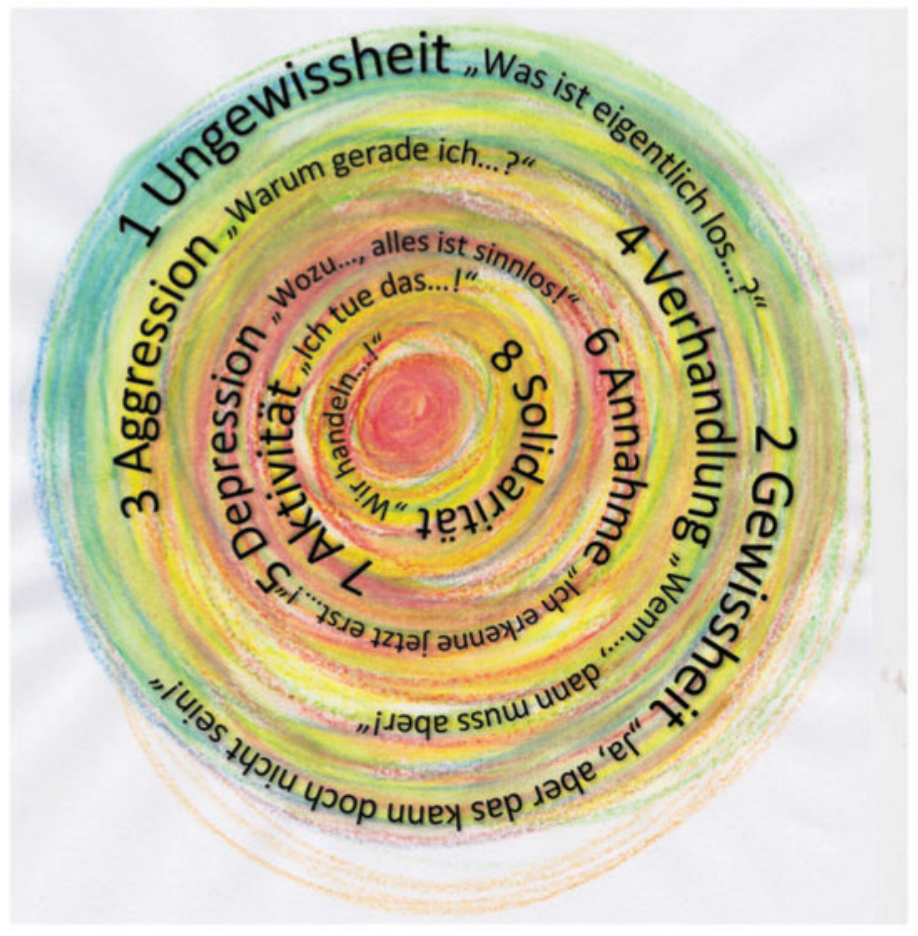

Abb. 2 Spiralphasenmodell nach Erika Schuchardt. (Illustration: Karin Weiß nach Schuchardt 2002 [19])
Führt man die Schwangerschaft weiter oder lässt man einen Abbruch vornehmen? Hier muss eine ausführliche, ergebnisoffene Beratung stattfinden, damit die Eltern eine informierte Entscheidung treffen können [23][20].

\section{PRAXIS}

Wichtige Marker für eine ergebnisoffene Beratung

- Einen geschützten Raum schaffen, um eine vertrauliche Gesprächssituation zu ermöglichen [8]

- Sinnvoll ist es, das Gespräch mit beiden Eltern zu führen, um mögliche Informationsverluste oder Verfälschungen zu vermeiden [20][8]

- Die emotionale Haltung, Empathie und Wertschätzung dem Kind gegenüber bleibt vorrangig im Gedächtnis der Eltern [8]

- Insbesondere Frauen, die das „Weiterführen der Schwangerschaft“ als selbstverständliche Entscheidungsoption vermittelt bekommen, fühlen sich in der Betreuung gut aufgehoben [17]

- Aktiv zuhören, bestätigen, ermutigen, Anerkennung ausdrücken

- Akzeptieren der eigenen Gefühle; Echtheit schafft Vertrauen und Sicherheit

- Offene, zugewandte Körperhaltung

- Aussagen des Paares nicht werten

- Offene Fragen fördern den Dialog

- Weitere Experten hinzuziehen [1]

\section{Begleitung nach der Diagnoseeröffnung}

Nach einer unerwünschten pränatalen Diagnose ist sehr schwierig zu erkennen, in welcher Phase die Eltern in der Verarbeitung dieser meist als traumatisch empfundenen Tatsache stehen. Sie kommen mit unterschiedlichen Lebenseinstellungen, Voraussetzungen und Bewältigungsmechanismen in diese Situation [8]. Es begleiten sie Schock, Unwissenheit, Unsicherheit und Abwehr [20]. Nicht immer fällt es uns leicht, die Eltern dort abzuholen, wo sie gerade stehen. Es ist wichtig, sich darüber im Klaren zu sein, dass die akute Belastungsreaktion sich in ganz unterschiedlicher Weise äußern kann [5]. Dabei sind alle persönlichen Reaktionen zu respektieren. Gerade im KlinikSetting können wir dabei Zeitdruck, Angst vor dem Mit-Aushalten und Überforderung verspüren. Ein Modell von Erika Schuchardt ( $\triangleright$ Abb.2) zeigt die einzelnen Krisenprozesse auf. Das Wissen über diese Spirale kann uns helfen, den Verarbeitungs- und Trauerprozess zu verstehen, zu erkennen und liebevoll zu begleiten.

Die Spirale im Krisenverarbeitungsmodell nach Erika Schuchardt veranschaulicht, dass jede Reaktion der Eltern ihren Sinn hat und dass diese Zeit und 
Anerkennung braucht. Unmittelbar nach dem Schock befinden sich die Eltern meist in Phase 1 oder 2. Fragen wie „Was soll das schon bedeuten?", „Hat das doch was zu bedeuten?“ oder „Das muss doch ein Irrtum sein?“ begleiten sie. Das darauffolgende Durchgangsstadium ist emotionaler. Es folgen Gefühlsausbrüche, Ablehnung, Verhandlungen und Sinnfragen. Jeder hat dabei sein eigenes Tempo. Es ist also kein festes Modell, welches eine Regel aufzeigt, wann man sich wo befinden muss. Die Qualität unserer Begleitung als Fachfrauen dieser Paare hat einen hohen Einfluss auf den oben aufgeführten Verarbeitungsprozess [19].

\section{PRAXIS}

Leitsätze für die empathische Begleitung der Eltern

\section{- Akzeptanz der Emotionen:}

- Für uns als Hebammen bedeutet dies: dabeibleiben, die Situation und Gefühle wie Ratlosigkeit, Enttäuschung, Abneigung und Wut mit aushalten, Gefühle stehenlassen und anerkennen.

\section{- Orientierungshilfe geben:}

- Offene und orientierungsgebende Gespräche führen und relevante Informationen geben. Das kann zu einem Perspektivenwechsel und zur Weiterentwicklung der eigenen Stärken führen [8].
- Bindungsstärkung unterstützen:

- Frauen im Gespräch immer wieder an die Bauchatmung erinnern. Dies schafft Wohlbefinden und Sicherheit.

- Entschleunigung schaffen:

- Durch ein verlangsamtes Tempo kann sowohl die Wahrnehmung des Gegenübers als auch die eigene Wahrnehmung gesteigert werden.

\section{Interdisziplinäres Unterstützungsnetzwerk}

Neben einer emphatischen und sensiblen Betreuung sollten wir gut informiert und vorbereitet sein [7]. Dazu zählt, Hilfsangebote und Beratungsstellen in der Region zu kennen, zu wissen, wie die Eltern mit Selbsthilfegruppen in Kontakt treten können oder wo sie finanzielle Hilfe oder Therapieangebote finden [16][22]. Ein interdisziplinäres Unterstützungsnetzwerk ist in jedem Fall genauso unerlässlich wie unser Mitgefühl und unser emotionaler Beistand [2]. Gespräche aller Art, vor allem mit Frauen in der gleichen Situation, gelten als sehr hilfreich [4].

Es gibt viele Informationen und Anlaufstellen, die Eltern auf das Leben mit einem kranken oder behinderten Kind vorbereiten, z. B. : 
- www.familienplanung.de/schwangerschaft/recht-undfinanzen/unterstuetzung-fuer-eltern-mit-behindertenkindern/

- www.bunter-kreis-deutschland.de

- www.bfvek.de vom Bundesverband zur Begleitung von Familien vorgeburtlich erkrankter Kinder e. V. (BFVEK)

- www.frühförderstellen.de

- www.kindergesundheit-info.de/themen/entwicklung/ behinderung/wegweiser-behinderung/

- www.emotionelle-erste-hilfe.org (emotionelle Unterstützung in Krisen und Bindungsförderung für Mutter und Kind)

\section{Unterstützung und Akzeptanz der elterlichen Entscheidung}

Egal, ob Eltern sich nun für die Weiterführung der Schwangerschaft oder für den Abbruch entscheiden, in gewisser Hinsicht ist es immer ein Verlust. Auch die Entscheidung, ein behindertes Kind zu bekommen ist quasi der Verlust von der Vorstellung des gesunden, des idealen Kindes. Sobald aber diese Entscheidung getroffen ist, erhoffen sich Eltern Akzeptanz und Unterstützung in dieser Entscheidung. Ab diesem Zeitpunkt ist Neutralität nunmehr unerwünscht [16] [17]. Jede Entscheidung, die die Frau trifft, ist richtig. Dass das vom Umfeld nicht immer so gesehen wird, ist bekannt. Wissen hat seinen Preis. Eltern müssen die Verantwortung tragen über Leben oder Tod eines eigentlich erwünschten Kindes. Wenn sich Eltern gegen die Fortführung der Schwangerschaft entscheiden, kann das Umfeld teilweise nicht verstehen, dass das Paar trauert, obwohl es doch selbst den Tod des Kindes beschlossen hat [13].

Auch bei der Fortsetzung der Schwangerschaft können Eltern ambivalente Gefühle verspüren [20]. Im Kopf und im Herzen muss Raum für ein besonderes Kind geschaffen werden. Das ist eine große Herausforderung [4]. Die Vorsorge sollte normal weitergeführt werden, eine gleichwertige Behandlung zu gesunden Schwangerschaften ist wichtig. Dennoch muss ein sensibler Umgang gepflegt werden, z. B. gilt es, das Mithören von fetalen Herztönen bei anderen Schwangeren möglichst zu vermeiden [17]. Auch Geburtsvorbereitung ist bedeutend und sinnvoll - meist wird eine Interaktion mit gesunden Schwangeren aber als schmerzlich empfunden, Kontakt will vermieden werden. Hier wäre das Angebot von Einzelgeburtsvorbereitung hilfreich [17][20]. Gleichzeitig bietet sich dabei die Gelegenheit, die Frau beim Zugang zu ihrem Kind zu unterstützen.

Wir wissen heute, dass „Nichtpassung“ zu Kontaktabbruch führt. Vorgeburtliche Erfahrungen werden tatsächlich im Körper des Ungeborenen gespeichert. Deshalb ist es auch wichtig, so weit wie möglich ein Maß an Sicherheit zu vermitteln [11], sei es durch
Meditationen, Fantasiereisen oder Entspannungsübungen. Auch ausgebildete Körpertherapeuten oder bindungsfördernde Eltern-Kind-Gruppen als Unterstützung wären hilfreich.

\section{Peripartale Betreuung}

Da es grundsätzlich ein breites Spektrum hinsichtlich der Art und Schwere der Fehlbildung bzw. Behinderung gibt, sind auch in der Betreuung während der Geburt viele Individualitäten möglich. Die Behandlungsmöglichkeiten hängen wesentlich von der erwarteten körperlichen Beeinträchtigung des Kindes ab. Zunächst muss eine Entscheidung über den Geburtsort und den Geburtsmodus getroffen werden [20]. Kinder mit Fehlbildungen, die mit dem Leben vereinbar sind, sind am besten in einem Perinatalzentrum aufgehoben, um dort intensivmedizinisch betreut zu werden. Wenn allerdings eine Letalität vorliegt, ist es durchaus möglich, auch eine außerklinische Geburt zu planen [5].

\section{Behandlung bei infauster Prognose}

Neben der Betreuung selbst liegt die Schwierigkeit im Spannungsfeld der Geburtsbegleitung, z. B. der technischen Überwachung oder dem Eingreifen bei kindlich indizierten Notfällen [17]. Für die Geburtsbegleitung bei infauster Prognose ist wichtig, zu wissen, was die Eltern sich wünschen. Möchten sie eine intensivmedizinische Versorgung oder lieber eine friedvolle Atmosphäre? Die Entscheidung über Notfallinterventionen muss im Vorfeld mit den Eltern besprochen werden. Die Empfindungen der Frauen gehen in dieser Hinsicht auseinander: Manche Frauen möchten technische Überwachung vermeiden, andere fordern sie ein, als etwas, das diesem Kind ebenso zusteht wie einem gesunden Kind [17]. Da es eine große Bandbreite möglicher Entscheidungsresultate gibt, sollten Vorstellungen, Wünsche und Bedürfnisse bestenfalls vorher in Gesprächen geklärt, schriftlich in Form eines Geburtsplans festgehalten werden und für alle Berufsgruppen einsehbar sein.

\section{Auf das Sterben vorbereiten}

Wenn sich die Eltern für eine Fortführung der Schwangerschaft bei einer letalen Anomalie entscheiden, haben sie in der Regel den Wunsch, ihr Kind lebend kennenzulernen. Es ist elementar für sie, ein Mitspracherecht zu haben. Sie möchten äußern können, welche Art der Versorgung sie für sinnvoll halten und wollen sich mit ihren Ängsten ernst genommen und handlungsmächtig fühlen [17]. Unabhängig davon, welchen Geburtsort und welchen Geburtsmodus die Frau wählt, ist das Gefühl der Geborgenheit und des Vertrauens zentral. Die Reduktion von Stressoren wie grelles Licht, Lärm oder Unruhe sind so bedeutend wie der ungestörte Körperkontakt-das Kind noch einmal lebend im Arm gehabt zu haben, zu wissen, dass es auf seinem kurzen und schweren Lebensweg nicht allein war, sondern seine 
Eltern bei sich hatte. Dem Kind beim Sterben nahe gewesen zu sein, ist später von unschätzbarem Wert für die früh verwaisten Eltern. Deshalb sollte es unbedingt in der Sterbephase bei ihnen sein [9].

Zur Erleichterung der Hausgeburtssituation ist es ratsam, ein Unterstützungsnetz zu organisieren: einen Arzt, der nach dem Tod des Kindes den Totenschein ausstellt, einen Kinderarzt, der beim Überleben des Kindes bei Bedarf Schmerzmittel verabreichen und es palliativ versorgen kann. [17]. So kann die Angst vor dem Leiden des Kindes gemildert werden.

\section{Geburt}

Während der Geburt ist das Schaffen einer höhlenartigen Atmosphäre unterstützend, in die sich die Gebärende zurückziehen kann: gedämpftes Licht, gelassene Stimmung und die Möglichkeit, verschiedene Gebärpositionen einzunehmen, z. B. durch einen Geburtshocker oder Partnersitz.

Die Geburt wird sehr unterschiedlich erlebt: Von Freude, das Kind endlich im Arm halten zu können, über Unsicherheit bis hin zu einem Gefühl von Bedrohung sind alle Emotionen möglich [17]. Teilweise haben die Pare keinen Geburtsvorbereitungskurs besucht, konnten keine Atemtechniken oder auch nicht den Umgang mit den Wehen erlernen. Besonders Frauen, deren Kind bereits präpartal verstorben ist, werden während der Geburt häufiger mit Gefühlen wie Einsamkeit konfrontiert [15]. Eine verstärkte emotionale Unterstützung unsererseits, kann den körperlichen und gefühlsbedingten Stress der Frau lindern und ihr Sicherheit gewährleisten.

\section{Schmerzmanagement}

Wie bei jeder anderen Geburt muss man auf die Bedürfnisse der Frau eingehen, diese erfragen und darf die Frau vor allem zu nichts drängen. Zwar erscheint es uns manchmal, als müssten wir gerade diese Frauen in ihrer Schmerzlinderung unterstützen, ihnen das Fühlen der Geburtswehen ersparen können-jedoch kommt dies nicht allen Gebärenden entgegen [13]. Offensichtlich wird der Geburtsschmerz als unzumutbarer wahrgenommen, als jener, der zur Geburt eines gesunden lebenden Kindes führt [15]. Doch die Selbstbestimmung bei der Geburtsarbeit ist wie bei „normalen Gebärenden“ enorm wichtig. Es braucht immer ein Gefühl von Verständnis, emotionaler Zuwendung und Einfühlsamkeit der Betreuungspersonen [17]. So sehen manche Frauen die Geburt immer noch als ein gemeinsam zu bewältigendes Ereignis und empfinden es so, als würden sie ihr Kind mit der Inanspruchnahme von Schmerzmedikation alleine lassen.

Falls allerdings eine Analgesie von der Frau gewünscht ist, sollte diese gut und ausreichend sein, allerdings sollten möglichst keine sedierenden Medikamente wie z. B. Diazepam oder Dolantin verabreicht werden, da diese 
das Einsetzen der Trauerarbeit behindern [13]. Besser Spasmolytika oder PDA anbieten.

Das Abnabeln kann durch den Vater erfolgen, wenn es die Situation erlaubt und er dies wünscht.

\section{Merke}

Bei früheren Schwangerschaftswochen ist zu beachten, dass der Verlauf, obwohl die Zervix zuvor unreif war, plötzlich rasch sein kann und der Muttermund nicht vollständig eröffnet sein muss. Bei Kindern, die bereits vor der Geburt verstorben sind, kommt es häufiger zu Einstellungsanomalien durch ihre fehlende Körperspannung. Aufrechte Geburtspositionen erscheinen hier günstig.

Das „normale Gebären“ sollte sich auch auf die Behandlung des Kindes beziehen, die Fehlbildung steht im Hintergrund-auch als Wertschätzung der Frau gegenüber, denn sie wird durch dieses Kind zur Mutter [17].

\section{Postpartale Betreuung}

\section{Das veränderte Bild}

Nach der Diagnose stellen sich Eltern oft eine Kreatur vor, die mit einem Baby nicht mehr viel gemeinsam hat, je nachdem wie es ihnen durch den Ultraschall beschrieben wurde. Das schürt Angst, sich dieses Wesen anzuschauen, gibt es doch Horrorvorstellungen in unterschiedlichem Ausmaß, die man sich aus den Informationen selbst zusammengesetzt hat. Wie wird es mit seinen Fehlbildungen aussehen? Sieht es noch aus wie ein Baby? Was ist mit seiner Hautbeschaffenheit, besonders, wenn es schon einige Tage zuvor gestorben ist? Überforderung und Scheu lassen sich erkennen. Eine Entfremdung zum eigenen Kind hat stattgefunden [17].

Postpartal sind die Eltern dann mit den Fehlbildungen direkt konfrontiert und müssen einen Umgang damit finden. Wir sollten versuchen, ihre Angst zu verstehen und ihnen ein emotionaler Beistand sein. So können wir ihnen eine Hilfestellung anbieten, wenn sie sich zunächst nicht trauen, ihr Kind zu sehen. Wir können es stellvertretend „mit ihren Augen“, für sie betrachten. Eine Möglichkeit ist, das Kind in ein Tuch oder eine Decke eingewickelt zu halten und den Eltern Stück für Stück jede Einzelheit liebevoll und anschaulich zu beschreiben [13]. Dabei kann man hervorragend Vergleiche zum Aussehen der Eltern herstellen und zum Beispiel die „kleine Stupsnase“ erwähnen, die es wohl von der Mutter hat, oder die langen Finger wie beim Vater. Auch die Andersartigkeit im Sinne sichtbar fehlgebildeter Körperteile darf hier benannt werden. Dennoch sollten wir sehr vorsichtig und behutsam sein, da es die Eltern zusätzlich frustrieren kann, dass sie nicht mit der gleichen liebevollen Art und Selbstverständlichkeit mit ihrem Kind umgehen können [8].

\section{PRAXIS}

\section{Anerkennung zeigen}

Frauen nehmen den Umgang der Hebamme mit ihrem Kind nach der Geburt genau wahr [17].

Gesten der Anerkennung sind zum Beispiel:

- Empathie zeigen

- Emotionale Betroffenheit wahrnehmen lassen

- Das Kind unbedingt behandeln, wie jedes andere „normale“ Baby; respektvoller Umgang

- Namentliches Ansprechen

- Liebevolles Handling

- Streicheln oder Halten des Kindes

- Sprechen mit dem Kind

- Gemeinsames Baden und Anziehen

- Lob und Anerkennung aussprechen

- Bei einem lebenden Kind, Eltern zur Geburt ihres Kindes beglückwünschen

„Man sieht nur mit dem Herzen gut. Das Wesentliche ist für die Augen unsichtbar."

\section{Antoine de Saint-Exupéry aus „Der kleine Prinz“}

\section{Umgang mit dem Kind}

Bei unserem Umgang mit dem Kind sollten wir uns immer bewusstmachen, dass Eltern ihr Kind nicht aus der klinischen Sicht des medizinischen Betreuungspersonals sehen, sondern mit den Augen des Herzens. Sie nehmen andere Details wahr [13]. Auch Kinder mit (schweren) Fehlbildungen tragen für die Eltern eine eigene Schönheit in sich [9].

Oft sind die Eltern darüber erleichtert, dass das Aussehen ihres Kindes nicht ihren schlimmsten Erwartungen entspricht [17] und suchen dann meist doch den Kontakt. Ob die Fehlbildungen dabei zunächst oder generell überdeckt werden sollen, spricht man mit den Eltern ab. Für dieses Stadium gibt es verschiedene Bewältigungsstrategien. Das Abwägen der eigenen Belastbarkeit steht im Vordergrund: Entweder die Eltern möchten das Kind unversehrt in Erinnerung behalten, oder andererseits sehen und verstehen, dass es eine Fehlbildung gibt, die nicht mit dem Leben vereinbar ist [17].

Die Suche nach ursächlichen Faktoren für die Fehlbildung, nach Gründen des Nicht-Überleben-Könnens, muss unbedingt ernstgenommen werden. Sie wird für die Mutter, die häufig mit Schuldgefühlen kämpft, als emotional vorteilhaft empfunden [15]. Ein Gespräch mit Spezialisten erweist sich hier als nützlich, denn es ist schwer zu akzeptieren, dass Fragen unbeantwortet bleiben [13]. 
Wenn die Eltern dennoch nicht bereit sind, ihr Kind zu sehen, ist auch das zu akzeptieren. Man muss ihnen diese Möglichkeit jedoch später immer noch einmal aufzeigen, denn oft haben sie nicht den Mut, aktiv danach zu fragen [13]. Die meisten Eltern, die ihr Kind nicht gesehen haben und so nicht Abschied von seinem Körper nehmen konnten, bereuen dies im Nachhinein zutiefst. Daher ist es wichtig, ihnen deutlich mitzuteilen, dass es nur diese kurze Zeitspanne gibt, um Erinnerungen zu sammeln, die für ein ganzes Leben reichen müssen [9].

\section{Namensgebung und religiöse Rituale}

Es ist immer gut, eine persönliche Anrede für das Kind zu verwenden, denn es hilft, ihm einen Platz in der Familie zu geben und es zu integrieren.

Für manche Paare ist es wichtig, dass ihr Kind noch getauft wird [17]. Wenn das Leben eines Neugeborenen bedroht ist, kann eine Nottaufe erfolgen. Diese kann von jedem Christen, also auch von einer nicht geistlichen Person, vorgenommen werden. So können auch Hebamme oder Arzt dies übernehmen. Das verwendete Wasser muss nicht geweiht sein, es kann einfach normales lauwarmes Leitungswasser als Taufwasser fungieren. Bei der Durchführung kann man folgenden Satz sprechen: „(Name des Kindes), ich taufe dich im Namen des Vaters und des Sohnes und des Heiligen Geistes. Amen." Dabei dreimal Wasser über die Stirn des Kindes laufen lassen. Danach kann ein Vaterunser gebetet werden. Voraussetzung für eine Taufe ist zum einen der Wunsch der Eltern und zum anderen, dass das Kind noch lebt. Wenn es bereits verstorben ist, kann nur noch eine Segnung stattfinden. Dazu kann man seine Hand auflegen und sprechen: „(Name des Kindes), es segne dich der Vater, der Sohn und der Heilige Geist." [9].

Bei anderen konfessionellen Zugehörigkeiten oder religiösen Orientierungen gibt es oft andere, sehr individuelle Vorstellungen. Hier kann es hilfreich sein, schon frühzeitig eine entsprechende Seelsorge zu konsultieren, die sich mit den jeweiligen Ritualen auskennt.

\section{Der bevorstehende Abschied}

Die verbleibende Zeit, die die Eltern mit ihrem Kind haben, ist sehr unterschiedlich. Bei den Eltern besteht ein Wunsch nach Anerkennung des Kindes und Anerkennung seiner Existenz. Die Wertschätzung für dieses Kind als Person unabhängig von seiner Fehlbildung ist enorm wichtig [17]. Man sollte ihnen Zeit lassen und auch das Alleinsein mit ihrem Kind anbieten.

Verschiedene Studien zeigen, dass es für Eltern essenziell ist, ihr Kind so lange bei sich haben zu dürfen, wie sie wollen [15][12]. Ein zu kurzer Zeitraum kann als belastend erlebt werden [17]. Sie möchten es sehen und halten. So erlangen sie die Bestätigung, dass es existiert und dass ihr 


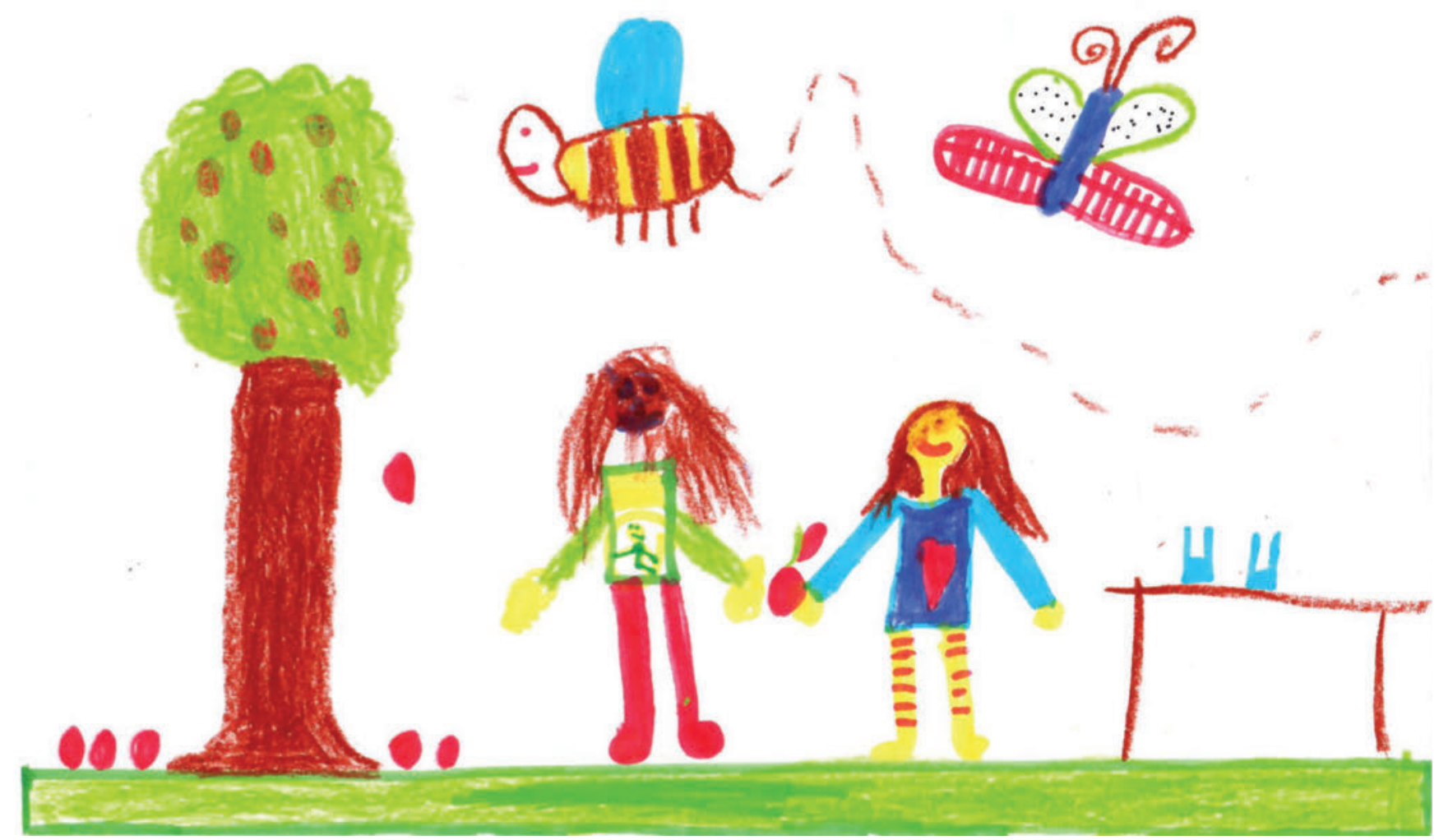

Abb. 3 Werdende Mutter trägt Kind mit diagnostizierter Fehlbildung im Bauch: Durch Malen können sich Kinder ausdrücken. Ihre Zeichnungen geben uns oft Aufschluss über Unbewältigtes [13]. (Quelle: privat)

Verlust greifbar und real ist [12]. Durch eine ausreichende Abschiedszeit kann der Tod begriffen werden. Die Eltern sollten unbedingt den Zeitpunkt des Abgebens des Kindes selbst bestimmen können. Wenn es gewünscht ist, sollte das Paar ihr Kind zu Hause verabschieden dürfen. Die Eltern müssen wissen, dass es durch den Bestatter die Möglichkeit gibt, das Kind von der Klinik nach Hause zu bringen [17][9]. Durch das Schaffen von Erinnerungen in den eigenen Wohnräumen kann die Existenz des Kindes an Realität gewinnen. Besonders in dieser Umgebung ist es leicht, die Geschwisterkinder miteinzubinden. Auch sie können vom Körper des Kindes Abschied nehmen.

\section{Geschwister ins Abschiednehmen einbeziehen}

Geschwisterkinder sollten nicht ausgeschlossen, sondern mit einbezogen werden. Sie können ansonsten keine Hilfe bei der Verarbeitung ihrer Gedanken und Gefühle bekommen. Ohnehin haben sie sehr feine Antennen und spüren an unseren Reaktionen, dass etwas nicht stimmt. Das kann dazu führen, dass sie eigene Fantasien darüber entwickeln, was wohl passiert sein mag [13]. Sie sind hochsensibel für die Emotionen ihrer Eltern - Sorgen, Ängste und Trauer bleiben nicht unbemerkt. Die Einbindung einer zusätzlichen Vertrauensperson aus dem Familienoder Freundeskreis erweist sich hierbei als hilfreich, um die Eltern psychisch zu entlasten. Auch Geschwister müssen die Möglichkeit bekommen, das neue Familienmitglied kennenzulernen und zu verabschieden [9]. Offene und ehrliche Kommunikation ist unabdingbar.

\section{Worte bewusst wählen}

Die Wortwahl sollte stets behutsam und für die Eltern verständlich sein. Fachtermini sind fehl am Platz. Unsere Sprache muss stets wertschätzend sein. Nach der Geburt wird ein Baby begrüßt-das steht auch einem toten Kind oder einem Kind mit Behinderung zu! Das Kennenlernen beinhaltet ein Stück Normalität [17].

\section{Frieden finden in einem liebevollen Abschied}

Wir können den Eltern die Trauer nicht abnehmen oder sie verringern, aber wir können gemeinsam mit ihnen den Schmerz aushalten [10].

Für den Trauerprozess ist es sinnvoll, den Eltern anzubieten, ihr Kind nach dem Versterben zu baden oder zu waschen. So wird ihnen die Übernahme einer 
elementaren elterlichen Aufgabe, nämlich der Körperpflege, ermöglicht [9]. Auch sollte man sie fragen, welche Kleidung sie für ihr Kind bevorzugen. Vielleicht möchten sie selbst etwas zum Anziehen oder eine Decke mitbringen, in die das Kind eingewickelt wird.

Weitere Informationen zum liebevollen Abschied eines Kindes sind hier zu finden:

- www.initiative-regenbogen.de

- www.veid.de vom Bundesverband Verwaiste Eltern und trauernde Geschwister in Deutschland (VEID)

- www.dein-sternenkind.eu

- www.balduins-box.de

- www.hopesangel.com

\section{Erinnerungen schaffen}

Wir müssen versuchen, eine Grundlage für eine wohltuende Erinnerung zu erlangen [15]. Elementar wichtig für die Eltern sind Beweise, die die Existenz des Kindes bestätigen [12]. Oft konnten durch seine kurze Anwesenheit auf der Welt nur wenige Personen das Kind kennenlernen. Daraus resultiert, dass auch nur wenige Menschen wissen und verstehen, um wen die Eltern trauern.

Fassbare Erinnerungen helfen, das Geschehene zu verarbeiten. So kann man zum Beispiel eine Erinnerungsbox gestalten und mit Gegenständen bestücken, die man mit dem Kind verbindet. Andenken, die einen positiven Einfluss auf den Trauerprozess haben, sind zum Beispiel Hand- und Fußabdrücke mit Stempelfarbe auf Pappe oder eine Haarsträhne vom Kind, welche allerdings nur mit ausdrücklicher Genehmigung der Eltern entnommen und dann am besten in einem kleinen Plastiktütchen aufbewahrt werden sollte.

Fotos halten genau fest, wie das Kind ausgesehen hat. So müssen sich die verwaisten Eltern später nicht ausschließlich auf ihre Erinnerung verlassen. Außerdem haben sie so die Möglichkeit auch anderen Personen ihr Kind zu zeigen und können ins Gespräch kommen, damit auch das Umfeld am Verlust Anteil nehmen kann [9].

Neben eigenen Bildern ist es empfehlenswert, den Eltern vorzuschlagen, einen entsprechend sensibilisierten Fotografen oder eine Fotografin zu beauftragen. Die Beschäftigten der Organisation „Dein Sternenkind“ sind z.B. ehrenamtlich tätig, den Eltern entstehen keinerlei Kosten. Sie bieten liebevolle und professionelle Erinnerungsfotos als Geschenk an. Die Eltern sollten möglichst frühzeitig über diese Möglichkeit informiert werden. Schön ist es, wenn sogar die Möglichkeit besteht, präpartal Vorstellungen und Wünsche zu besprechen. Aufnahmen des Neugeborenen mit seinen Geschwistern, auf dem Arm von den Großeltern oder sonstige Motive können den Eltern ein Anliegen sein.

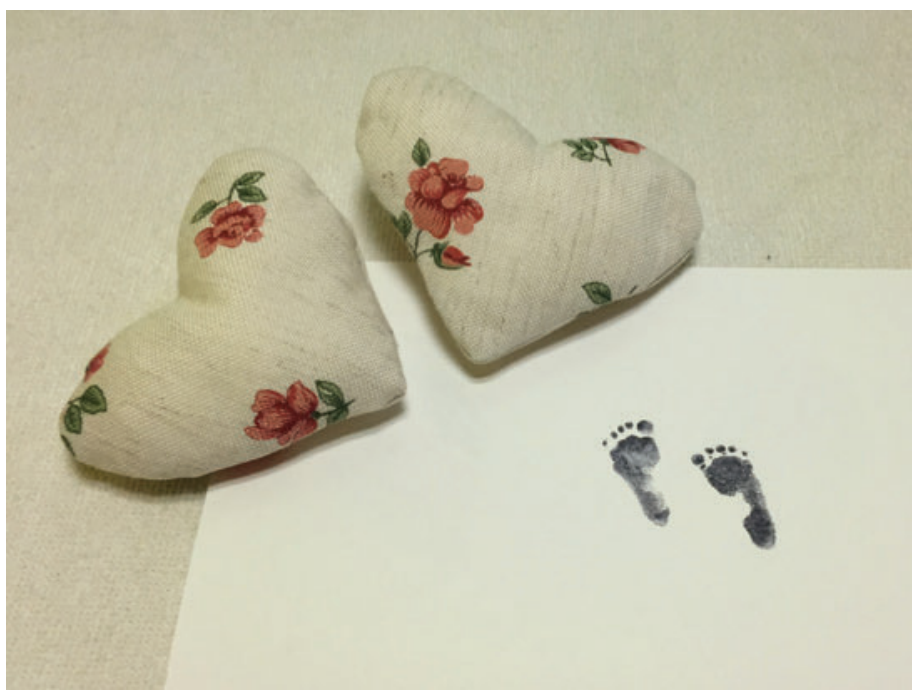

- Abb. 4 Erinnerungsstücke wie diese helfen bei der Trauerverarbeitung: Von den beiden identischen Stoffherzen verbleibt das eine beim Kind, das andere erhalten die Eltern. (Foto: Jelena Rensinghoff)

Einige Eltern haben erst später den Wunsch, sich Fotos anzuschauen. Für diesen Fall ist wichtig, ihnen Fotos in einem verschlossenen Umschlag mitzugeben oder mit Hinweis an die Eltern in der Akte aufzubewahren.

\section{PRAXIS}

Fassbare Erinnerungen gestalten

- Hand- und Fußabdrücke

- Namensbändchen

- Namenskarte mit Geburtsdaten (Name, Geburtstag und -stunde, Gewicht, Länge)

- Haarlocke (nur mit elterlicher Genehmigung!)

- Brief an das Kind

- Zeitung/Wetterbericht vom Geburtstag

- Kiste selbst gestalten

- zwei identische Kuscheltiere o. ä. - eines für die Eltern, eines mit zum Kind geben

- Decke oder Tuch, in die das Kind eingewickelt war

- Nabelklemme (beim Kind durch ein Bändchen aus Stoff oder durch einen Wollfaden ersetzen)

- Fotos

\section{Auf das unerwartete Überleben des Kindes vorbereitet sein}

Die Prognose bezüglich einer Überlebenswahrscheinlichkeit kann auch bei schweren Fehlbildungen (z.B. Nierendysplasien) nicht präzise vorhergesagt werden. Dann kann es sein, dass im Kreißsaal innerhalb kürzester Zeit von Palliativversorgung auf den maximalen Einsatz lebenserhaltender Maßnahmen umgeschwenkt werden muss [9]. 
Eine unerwartete und besondere Herausforderung ist das Überleben des Kindes bei einer pränatal infausten Prognose. In einer solchen Situation beginnt bei den Eltern unmittelbar das Gedanken- und Gefühlskarussell, wie im Spiralenmodell ( $\mathbf{A} \mathbf{b b} \mathbf{2}$ ) beschrieben. Sowohl für das Paar als auch für die Begleiter fehlt vorerst die Orientierung. Es folgen viele Entscheidungssituationen. Häufig werden Entscheidungen dann überstürzt getroffen. Die gesamte Bandbreite an Emotionen kann in diesen Situationen auftreten, unter anderem Überforderung, Informationsbedürfnis, Gefühl des Alleinseins, Freude und Euphorie, aber auch Gefühllosigkeit und Schock. Eine Absprache mit den Eltern und auch die interdisziplinäre Kommunikation gestaltet sich in diesem Fall als sehr schwierig. Es stellt sich die Frage, wie die Hebamme hier adäquat reagieren kann und welche Standards und Methoden sich anbieten, um den Eltern in dieser Situation bestmöglich Orientierung und Sicherheit zu geben.

In der Schock-Phase wird der Dialog zu sich selbst abgebrochen. Der Mensch spürt sich nicht mehr, kann nicht mehr beschreiben, was im eigenen Körper passiert [11]. Dies führt zu einer Art Versteinerung, wodurch die Eltern sich abgrenzen und aus der Situation zurückziehen. Auf diese Weise kommt es häufig auch zu einer Ablehnung des Neugeborenen [17]. Für die Fachfrauen ist es sehr wichtig, nicht über diese scheinbare Emotionslosigkeit zu urteilen und kein bestimmtes Elternverhalten zu erwarten. Wichtig ist, das Dabeibleiben, das Informieren und das Anerkennen dieser Emotionen.

Überlebt das Kind deutlich länger als prognostiziert, können sich die Emotionen der Eltern im Lauf der Überlebenszeit des Kindes verändern. Bei manchen Paaren zeigt sich eine Ambivalenz ihrer Gefühle. Der Wunsch nach dem Überleben des Kindes und die Angst vor seinem Sterben stehen der Angst vor dem Überleben des Kindes gegenüber. Diese ambivalenten Gefühle lösen Schuldgefühle aus [17]. Für die Fachpersonen ist es daher besonders wichtig, sich diese ambivalenten Gefühle der Eltern bewusst zu machen und nicht zu bewerten. Den Eltern muss Raum für ihre Gefühle gegeben werden, um die Voraussetzung für ein gutes Vertrauensverhältnis zu schaffen. Dies kann durch gute Orientierung, Einbindung, Entlastung und Zeit unterstützt werden. Wir müssen Standpunkte ernst nehmen, Gespräche auf Augenhöhe führen und, wenn nötig, eine Entschuldigung einräumen [17].

„Das eine Kind ist so, das andre Kind ist so, doch jedes Kind ist irgendwann geboren irgendwo."

Rolf Zuckowski, Liedtext

\section{Betreuung im Wochenbett}

Wenn wir als Hebammen Familien begleiten, deren Geschichte auch uns viel Kraft kostet, ist es ratsam, diese Besuche ans Ende unserer Route für die Wochenbettbesuche zu legen. Nur so können wir in der empathischen Betreuung echt sein und müssen uns bei den Besuchen der nachfolgenden Familien nicht verstellen. Wir sollten unsere emotionalen Reaktionen nicht unterschätzen.

\section{Selbstfürsorge}

Auch für die Hebamme kann eine solche Begleitung emotional belastend sein. Deshalb ist es unerlässlich, auf eine gute Selbstpflege zu achten. Diese beinhaltet u.a. die Wahrnehmung der persönlichen Grenzen und eigener körperlicher Warnsignale. Es ist absolut in Ordnung und auch notwendig, sich Schwäche zuzugestehen.

Die Belastung ist stärker, je unvorbereiteter die Hebamme ist. Hilfreich ist das Lesen von Büchern und die Auseinandersetzung mit dem Thema. Fortbildungen stärken die Sicherheit im Umgang mit den Eltern und helfen, die richtigen Worte zu finden [10][14].

Eine gemeinsame Fallbesprechung mit allen teilnehmenden Akteuren ist neben einer Supervision ideal. Hier können persönliche Grenzen, belastende Situationen und die eigene Überwältigung mit passenden Hilfsstrategien oder -angeboten beleuchtet werden.

\section{FAZIT}

Gibt es nun den richtigen Weg? Durch die intensive Auseinandersetzung mit diesem Thema sind wir zu dem Schluss gekommen, dass existenzielle Entscheidungen, die eine derartige Bedeutung haben, selten zu einem Wohlbefinden führen. Somit gibt es in dem Prozess kein richtig und kein falsch. Unsere Aufgabe als Hebammen ist es, auf dem individuellen Weg der Eltern mit- und nicht voranzugehen. Sie müssen darauf vertrauen können, alles sagen zu dürfen, was sie beschäftigt, und dennoch von uns unterstützt, respektiert und wertgeschätzt zu werden. Denn ob eine Person gestärkt oder geschwächt aus einer Lebenskrise hervorgeht und diese verarbeiten kann, hängt auch wesentlich von unserer Betreuung und Begleitung ab.

\section{Autorinnen / Autoren}

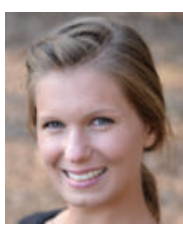

Jelena Rensinghoff ist frei praktizierende Hebamme an der Abteilung für Geburtshilfe und Pränatalmedizin, Klinik Hallerwiese, in Nürnberg. Sie studiert aktuell im Masterstudiengang Advanced Practice Midwifery. 


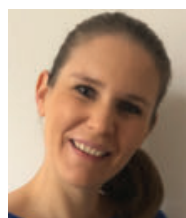

Karin Weiß ist frei praktizierende Hebamme an der Abteilung für Geburtshilfe und Pränatalmedizin, Klinik Hallerwiese, in Nürnberg. Sie beschäftigt sich mit Emotioneller Erster Hilfe und absolviert aktuell eine Ausbildung zur EEHFachberaterin.

\section{Korrespondenzadresse}

Klinik Hallerwiese

DIAKONEO

Abteilung für Geburtshilfe und Pränatalmedizin

Jelena Rensinghoff

St.-Johannis-Mühlgasse 19

90419 Nürnberg

E-Mail: Jelli-Rensinghoff@web.de

\section{Literatur}

[1] Ahrendt C. Hebammenrolle, Kommunikation und pädagogische Hebammenaufgaben. In: Stiefel A, Hrsg. Hebammenkunde. Stuttgart: Hippokrates 2013; 33-57

[2] Asplin N, Wessel H, Marions L et al. Pregnancy termination due to fetal anomaly: Women's reactions, satisfaction and experiences of care. Midwifery 2014; 30: 620-627

[3] Baldus M. Von der Diagnose zur Entscheidung. Eine Analyse von Entscheidungsprozessen für das Austragen der Schwangerschaft nach der pränatalen Diagnose Down-Syndrom. Bad Heilbrunn: Klinkhardt 2006

[4] Bundeszentrale für gesundheitliche Aufklärung (BZgA). Besondere Umstände - Informationen nach einem auffälligen pränataldiagnostischen Befund. Köln: BZgA 2018

[5] Bundeszentrale für gesundheitliche Aufklärung (BZgA). Pränataldiagnostik - ein Handbuch für Fachkräfte aus Medizin und Beratung. Köln: BzgA 2010

[6] Bundeszentrale für gesundheitliche Aufklärung (BZgA). Schwangerschaftserleben und Pränataldiagnostik-Repräsentative Befragung Schwangerer zum Thema Pränataldiagnostik. Köln: BZgA 2006

[7] Chaplin J, Schweitzer R, Perkoulidis S. Experiences of prenatal diagnosis of spina bifida or hydrocephalus in parents who decide to continue with their pregnancy. Journal of Genetic Counseling 2005; 14: 151-162

[8] Christ-Steckhan C. Elternberatung in der Neonatologie. München: Reinhardt 2005

[9] Garten L, von der Hude K. Palliativversorgung und Trauerbegleitung in der Neonatologie. Berlin, Heidelberg: Springer 2014
[10] Harder U. Wochenbettbetreuung in der Klinik und zu Hause. Stuttgart: Hippokrates 2011; 174-184

[11] Harms T. Emotionelle Erste Hilfe. Berlin: Ulrich Leutner 2008

[12] Jones K, Baird K, Fenwick J. Women's experiences of labour and birth when having a termination of pregnancy for fetal abnormality in the second trimester of pregnancy. Midwifery 2017: 50: 42-54

[13] Lothrop H. Gute Hoffnung, jähes Ende. München: Kösel 2016

[14] Martinez-Serrano P, Palmar-Santos AM, Solis-Munoz M et al. Midwives' experience of delivery care in late foetal death: A qualitative study. Midwifery 2018; 66: 127-133

[15] Rådestad I, Nordin C, Steineck G et al. A comparison of women's memories of care during pregnancy, labour and delivery after stillbirth or live birth. Midwifery 1998; 14: 111-117

[16] Redlinger-Grosse K, Bernhardt BA, Berg K et al. The decision to continue: The experiences and needs of parents who receive a prenatal diagnosis of holoprosencephaly. American Journal of Medical Genetics 2002; 112: 369-378

[17] Rost K. Wenn ein Kind nicht lebensfähig ist. Osnabrück: Universitätsverlag 2015

[18] Santalahti P, Hemminki E, Latikka AM et al. Women's decision-making in prenatal screening. Social Science and Medicine 1998; 46: 1067-1076

[19] Schuchardt E. Warum gerade ich? Leben lernen in Krisen. Göttingen: Vandenhoeck \& Ruprecht 2002

[20] Statham H, Solomou W, Chitty L. Prenatal diagnosis of fetal abnormality: Psychological effects on women in low-risk pregnancies. Bailliere's Clinical Obstetrics and Gynaecology 2000; 14: 731-747

[21] Wassermann K, Rhode A. Pränataldiagnostik und psychosoziale Beratung: Aus der Praxis für die Praxis. Stuttgart: Schattauer 2009

[22] Wilken E. Pränatale Diagnostik, Schwangerschaftskonfliktberatung und Bewertung behinderten Lebens. In: Wilken $\mathrm{U}$, Thole W, Hrsg. Kulturen Sozialer Arbeit. Wiesbaden: Springer 2010; 135-146

[23] Zimmermann R, Gembruch U.Schwangerschaftsvorsorge. In: Schneider H, Husslein P, Schneider K-TM, Hrsg. Die Geburtshilfe. Berlin Heidelberg: Springer 2016

Bibliografie

DOI https://doi.org/10.1055/a-1029-5610

Die Hebamme 2019; 32: 25-35

(c) Georg Thieme Verlag KG Stuttgart · New York ISSN 0932-8122 